\section{UNILATERAL THORACOSCOPIC SURGICAL APPROACH FOR DIFFUSE EMPHYSEMA}

We evaluated the use of a lateral thoracoscopic approach for lung reduction surgery in patients with diffuse emphysema. Sixty-seven patients with a mean age of 61.9 years underwent operation. Operative side was determined by preoperative imaging. The procedures were laser ablation in $\mathbf{1 0}$ patients and stapler resection in 57 patients. Ten patients, including six of the 10 patients in the laser-only group had poor outcome (death or hospitalization longer than 30 days), leading us to abandon the laser technique. Of the remaining 57 patients undergoing primary stapled resection, duration of chest tube placement averaged 13 days (range 3 to 53 days) with a mean hospital stay of 17 days (range 6 to 99 days). Seven patients required ventilation for longer than 72 hours, six patients underwent conversion of the procedure to open thoracotomy, four patients acquired arrhythmias, and three patients were treated for empyema. There was one early death $(1.7 \%)$, from cardiopulmonary failure. Forty patients returned for 3-month evaluation. Significant $(p<0.0001)$ improvements were seen in forced vital capacity $(2.69 \mathrm{~L}$ after vs $2.26 \mathrm{~L}$ before) and forced expiration volume in 1 second (1.04 $\mathrm{L}$ after vs $0.82 \mathrm{~L}$ before), with 25 of 40 patients $(63 \%)$ showing an improvement of more than $20 \%$. Lung volume measures, in particular residual volume, fell significantly. Arterial blood gas analysis revealed that carbon dioxide tension fell significantly in patients with preoperative hypercapnia (carbon dioxide tension $>45 \mathrm{~mm}$ $\mathrm{Hg}, p=0.018$ ). Six-minute walk test results improved (894 feet after vs 784 feet before, $p=0.002$ ), and symptomatic benefit was confirmed by significant improvement in the dyspnea index. The combination of both hypercapnia and reduced single-breath diffusing capacity for carbon monoxide was significantly more frequent $(p=0.0026)$ and was $86 \%$ specific (5 of 6 patients) in predicting serious postoperative risk. We conclude that the lateral thoracoscopic surgical approach to diffuse emphysema offers significant improvement in pulmonary mechanics and functional impairment. Patients with a combination of hypercapnia and reduced single-breath diffusing capacity for carbon monoxide should not be considered for this procedure because of significant perioperative risk. (J THORAC CARDIOVASC SURG 1996;111:308-16)

Robert J. Keenan, $\mathrm{MD}^{\mathrm{a}}$ (by invitation),

Rodney J. Landreneau, MD, ${ }^{a}$ Frank C. Sciurba, MD $^{\mathrm{b}}$ (by invitation), Peter F. Ferson, $\mathrm{MD}^{\mathrm{a}}$ (by invitation), J. Michael Holbert, $\mathrm{MD}^{\mathrm{c}}$ (by invitation), Manuel L. Brown, $\mathrm{MD}^{\mathrm{c}}$ (by invitation), Lynda S. Fetterman, $\mathrm{RN}^{\mathrm{a}}$ (by invitation), and Claudia M. Bowers, BSN, $\mathrm{MSc}^{\mathrm{a}}$ (by invitation), Pittsburgh, $\mathrm{Pa}$.
From the Divisions of Cardiothoracic Surgery ${ }^{\mathrm{a}}$ and Pulmonary Medicine ${ }^{b}$ and the Department of Diagnostic Imaging, ${ }^{c}$ University of Pittsburgh.

Read at the Seventy-fifth Annual Meeting of The American Association for Thoracic Surgery, Boston, Mass., April 23-26, 1995.

Address for reprints: Robert J. Keenan, MD, Suite 300, 3471 Fifth Ave., Pittsburgh, PA 15213.

Copyright (C) 1996 by Mosby-Year Book, Inc.

0022-5223/96 $\$ 5.00+0 \quad \mathbf{1 2 / 6 / 6 8 9 1 9}$
Chronic obstructive lung disease is America's number-three killer, accounting for one in seven deaths. In 1990, the National Health Interview Survey estimated the nationwide prevalence of emphysema at 2 million cases. More than $42 \%$ of persons with emphysema have limitations in activities of daily living. ${ }^{1}$ The progression of emphysema is insidious, and severe symptoms may not develop for many years. Most patients die within 2 years 
after medical treatment has become ineffective. Lung transplantation can help only a relative few, and many patients with emphysema are beyond the age limits for transplantation candidacy.

Excision of large bullae, occupying at least one third of the volume of the affected lung, is well established for relief of compression causing dyspnea. ${ }^{2}$ More recently, however, attention has focused on the potential benefits of surgical resection of diffusely emphysematous tissue in an effort to reduce dyspnea and improve exercise tolerance. This effort is based on the pioneering work of Brantigan, Kress, and Mueller, ${ }^{3}$ who postulated that denervation of the lung and removal of wedges of diseased tissue to reduce the overall volume of the lung and reshape its configuration would improve the elastic recoil of small airways and improve diaphragmatic excursion. This early experiment was successful in some patients, but the considerable morbidity associated with the standard thoracotomy technique led to abandonment of the procedure.

Video-assisted thoracic surgery has been increasingly used for a number of pulmonary surgical interventions. This approach, which avoids the necessity for thoracotomy, has significantly reduced morbidity otherwise accompanying surgical intervention for selected pulmonary problems. ${ }^{4}$ Use of video-assisted thorascopic laser ablation to treat bullous emphysema has been reported. ${ }^{5}$ Wakabayashi and coworkers ${ }^{6}$ first described its use for diffuse emphysema in a small group of patients, although the perioperative complication rate was significant. ${ }^{7}$ Results from this series indicated subjective relief of symptoms in most patients and significant improvement in a small subgroup with respect to pulmonary function and exercise tolerance. More recently, Cooper and associates ${ }^{8}$ reported on 20 patients who, after median sternotomy and stapled resection of emphysematous tissue, had improvements in pulmonary function and symptoms, with an average increase in forced expiratory volume in 1 second $\left(\mathrm{FEV}_{1}\right)$ of $82 \%$ over preoperative values.

We undertook this study to evaluate the efficacy of a unilateral thoracoscopic approach to lung reduction surgery. The aims of the study were to determine the effectiveness of thoracoscopic laser and stapled ablation in improving pulmonary function and reducing symptoms, and to investigate the physiologic mechanisms by which this improvement is achieved. A further aim was to evaluate the prognostic value of preoperative physiologic and anatomic selection factors.

\section{Methods}

Patient population. Sixty-seven adults with diffuse emphysema were enrolled in this study. The participants all had end-stage emphysema, a severely impaired quality of life despite maximal medical therapy, and disabling dyspnea at less than 50 yards of walking. Patients with bullous emphysema whose disease was characterized by large bullae with compressive signs on computed tomographic (CT) scan were excluded from the study. Fourteen candidates had been evaluated by the lung transplantation program and were offered lung reduction surgery as an alternative or as a bridge to transplantation. All patients were receiving maximal bronchodilator therapy, with 18 subjects also taking regular doses of prednisone. Supplemental oxygen therapy was required by $80 \%$ of our candidates, with $62 \%$ of all patients using oxygen continuously.

Exclusion criteria included known pulmonary hypertension (systolic pulmonary arterial pressure $>60 \mathrm{~mm} \mathrm{Hg}$ ), smoking of tobacco products within the last 3 months, morbid obesity ( $>1.5$ lean body weight), unstable coronary artery disease, end-stage cancer, nonambulatory status, and ventilator-dependence. Patients who had previously undergone open thoracic surgical procedures on the side designated for lung reduction were also excluded. Subjects who had undergone previous contralateral thoracic surgery or cardiac procedures through a median sternotomy remained eligible for the unilateral thoracoscopic approach.

All patients were seen in consultation by the nutrition service for assessment of nutritional status and recommendations for supplementation. Patients who were taking more than $20 \mathrm{mg} /$ day of prednisone were not eligible for operation until they were weaned below this level. All patients were encouraged to participate in a cardiopulmonary rehabilitation program for a minimum of 2 months, but this was not a requirement of the program.

The study was approved by the University of Pittsburgh Institutional Review Board for Biomedical Research.

Pulmonary evaluation. Standard preoperative studies to evaluate patients with poor pulmonary reserve were performed. Testing was conducted 1 to 3 weeks before operation. These studies included a preoperative electrocardiogram, routine blood work (arterial blood gases; complete blood cell count; serum chemistry profile; and coagulation profile including prothrombin time, partial thromboplastin time, thromboplastin time, and platelets), posterior-anterior and lateral chest roentgenograms, and two-dimensional echocardiography to assess global ventricular function.

Standard pulmonary function tests including spirometry to measure actual, predicted, and percentage of predicted forced vital capacity (FVC), $\mathrm{FEV}_{1}, \mathrm{FEV}_{1} / \mathrm{FVC}$ ratio, forced expiratory flow at $25 \%$ to $75 \%$ of FVC, peak expiratory flow, and maximal voluntary ventilation were conducted and the results were recorded. ${ }^{9}$ The singlebreath diffusing capacity for carbon monoxide (DLCO) and maximal respiratory pressures were also deter- 
Table I. Assessment of functional impairment with dyspnea indexes

\begin{tabular}{ll}
\hline Baseline dyspnea index (BDI) \\
Functional impairment \\
No impairment. Able to carry out usual \\
activities and occupation without short- \\
ness of breath. \\
Slight impairment. Distinct impairment in \\
at least one activity but no activities \\
completely abandoned. Reduction in \\
activity at work or in usual activities \\
seems slight or not clearly caused by \\
shortness of breath. \\
Moderate impairment. Patient has \\
changed jobs and/or abandoned at least \\
one usual activity because of shortness \\
of breath. \\
Severe impairment. Patient unable to \\
work or has given up most or all usual \\
activities because of shortness of \\
breath. \\
Very severe impairment. Unable to work \\
and has given up most or all usual ac- \\
tivities because of shortness of breath. \\
Grade 13 \\
Grade 0
\end{tabular}

mined. ${ }^{10}$ Body plethysmography was used to measure thoracic gas volumes functional reserve capacity, total lung capacity (TLC), and residual volume. ${ }^{11}$

CT scans of the chest were performed with $10 \mathrm{~mm}$ slices obtained every $10 \mathrm{~mm}$ with a GE HiSpeed Advantage scanner (General Electric CGR USA, Milwaukee, Wis.). Images were filmed at a window of 1500 and a level of -600 . Density mask images were obtained by highlighting voxels that corresponded to -910 to -1024 Hounsfield units with a standard GE density mask program. This range has been shown to correspond to emphysema in pathologic specimens ${ }^{12}$ and with estimates of pulmonary function. Ventilation and perfusion scans were obtained in which segmental quantitation of ventilation and perfusion were calculated to establish baseline function. Single photon-emission CT (SPECT) perfusion imaging was performed with a dual-head gamma camera. The studies were obtained in a 128 matrix in word mode. A 1.6 acquisition zoom at 3 degrees of angular sampling with a noncircular orbit for 20 minutes was used. The data were reconstructed by means of a Hanning filter with a cutoff frequency of $1.3 \mathrm{cycles} / \mathrm{cm}$ and axial smoothing. The transaxial data were reprojected into 36 frames by means of volume-rendering with a linear maximal value technique. ${ }^{14}$ Images were viewed in a rotating display mode. This three-dimensional image of the lungs assisted in determining the areas to be resected.

Exercise capacity was tested with a 6-minute walk monitored by pulse oximetry. Supplemental oxygen was titrated before the testing maneuver to determine a flow rate that would maintain oxygen saturation above $90 \%$ during usual pace exertion. Patients were given a standard set of instructions before the maneuver, and no coaching was provided during the testing.

The degree of functional impairment from dyspnea was determined by use of the baseline dyspnea index. ${ }^{15}$ One of the investigators selected from one to five grades of dyspnea on the basis of an extensive history (Table I).

Three months after operation, pulmonary function, diagnostic imaging, and the 6-minute walk were repeated to evaluate the extent of improvement and to determine the mechanisms involved. Patients and technicians were blinded to the preoperative values during postoperative testing. Changes in the degree of functional impairment after surgery was determined at the 3-month study point by means of a transitional dyspnea index (TDI). A TDI score, ranging from -3 (major deterioration) to +3 (major improvement), was assigned after an extensive interview (Table I).

Operative procedure. Electrocardiographic monitoring, radial arterial cannulation, and central venous access for monitoring of pulmonary artery pressures and cardiac output were routine in all cases. An epidural catheter was placed in a low thoracic position to provide postoperative analgesia and also to augment the intraoperative anesthetic management. This strategy was aimed at reducing inhalational anesthetic and systemic narcotic requirements during the procedure.

Selective airway intubation was accomplished with a left-sided double-lumen endotracheal tube. Preparation for high-frequency jet ventilation was made in case that ventilatory method were to become necessary during the lung reductive procedure. Pulse oximetry and end-tidal carbon dioxide monitoring were followed throughout the procedure. Baseline arterial blood gas values and cardiac output were obtained before the incision. 


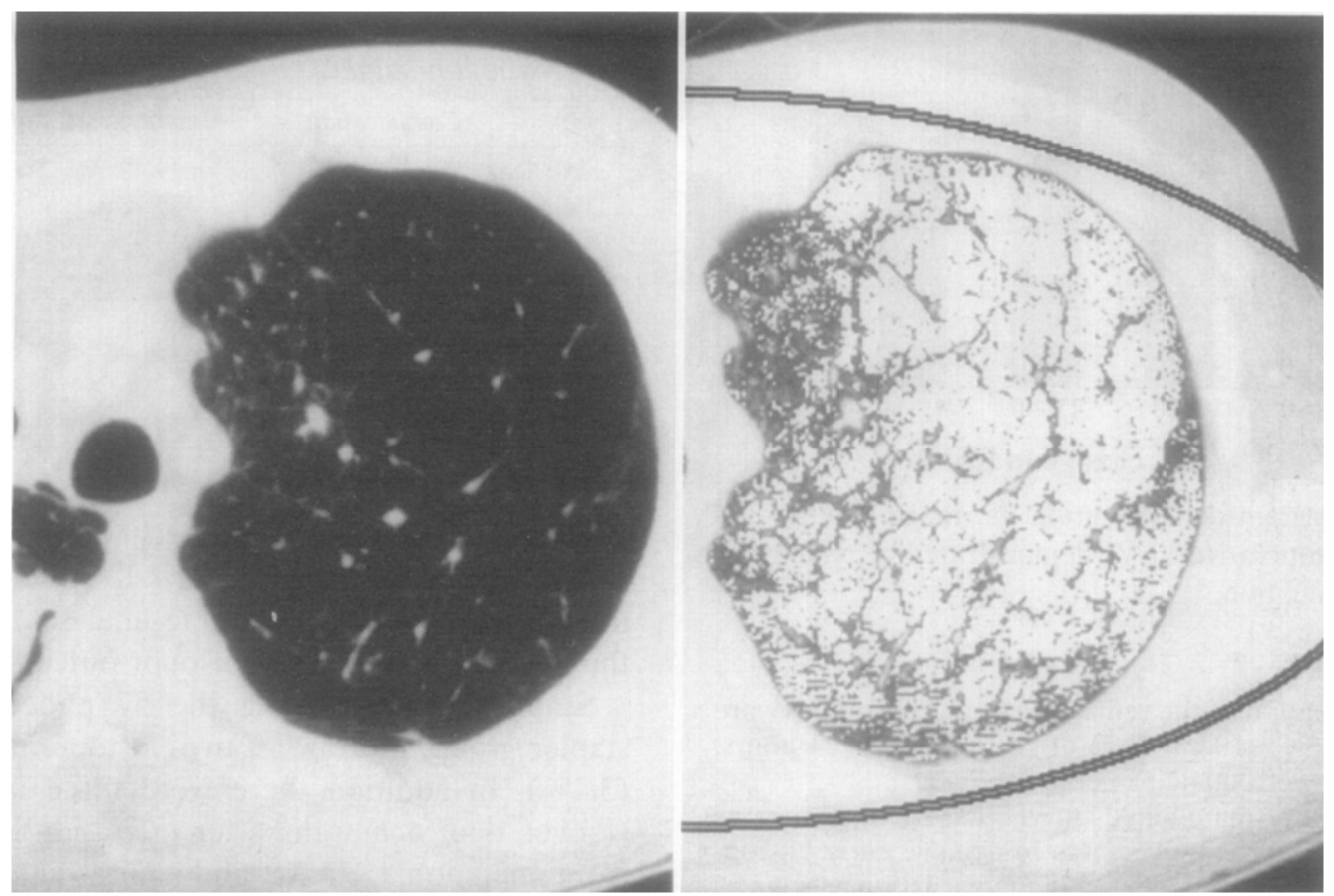

Fig. 1. CT images, as normally generated (left) and with density mask imaging (right) to demonstrate more clearly the areas of anatomic emphysema (shown in white).

Lung reduction was performed on the lung most severely affected by the emphysematous process, as determined roentgenographically (by chest radiograph, CT scan, and ventilation and SPECT perfusion nuclear scintigraphy). The areas of the lung to be resected during the lung reduction procedure were also primarily determined by the preoperative roentgenographic findings. In particular, the density mask images of the CT scan highlighted the areas of worst anatomic disease, as well as zones of more normal lung (Fig. 1), whereas the SPECT perfusion study demonstrated zones of significant hypoperfusion. These areas were focused on during the resection, particularly when the ventilation studies had shown associated washout abnormalities.

The patient was placed in a full lateral decubitus position. A three- to five-port intercostal access technique was used in most circumstances. The access for the "operative" thoracoscope was achieved in the seventh to eighth intercostal space at the mid to posterior axillary line. After intercostal access for the thoracoscope was established, subsequent sites of access for instrumentation were achieved in the sixth or seventh interspace in the posterior axillary line and in the seventh interspace in the anterior axillary line.

In 10 patients, laser ablation of emphysematous tissue was the sole modality of lung reduction. In these patients, the areas of significant disease were diffusely scarified with the neodymium:yttrium-aluminum-garnet (Nd:YAG) laser, set at 10 to 15 watts in the noncontact mode. Endoscopic stapler resection of portions of the lung became the preferred approach, with either a $30 \mathrm{~mm}$ (United States Surgical Corp., Norwalk, Conn.) or a 60 mm (Ethicon Endo-Surgery, Cincinnati, Ohio) stapler used. Areas of bullous formation located near the pulmonary hilum or at angles difficult to reach with the endoscopic stapler were managed with Nd:YAG laser bullous ablation. Similarly, areas of "vanishing lung," consolidated areas of numerous bullae, were initially scarified and reduced with laser energy before stapler application. In practice, about $90 \%$ of the lung reduction in any patient was accomplished with the endoscopic stapler, with the noncontact Nd:YAG laser being required sparingly. The lung resection was aimed at reducing the overall volume of the lung by $25 \%$. Strips of lung tissue were resected along the leading edges of each lobe, such as along the fissures or anteriorly and posteriorly to the apex of the upper lobe and along the basal segments or superior segment of the lower lobe. The extent of resection in each area was determined in advance by the results of the SPECT perfusion studies. The lung was partially inflated periodically during the procedure to estimate the extent of resection accomplished in each lobe of the lung and thus gauge the progress of the procedure to avoid overresection.

At the termination of the procedure, three chest tubes were strategically placed into the pleural cavity through the intercostal access used during the operative intervention. Two tubes were positioned at the apex, and a single right-angle tube was placed in the costophrenic angle. The lung was gently reexpanded and ventilation was reestablished, with the aim of keeping peak airway pressures at the minimum required to achieve an adequate tidal volume (less than $30 \mathrm{~mm} \mathrm{Hg}$ ). Air leakage from the chest tubes was assessed before the surgical field was taken down. To avoid an excessive pressure gradient and possi- 


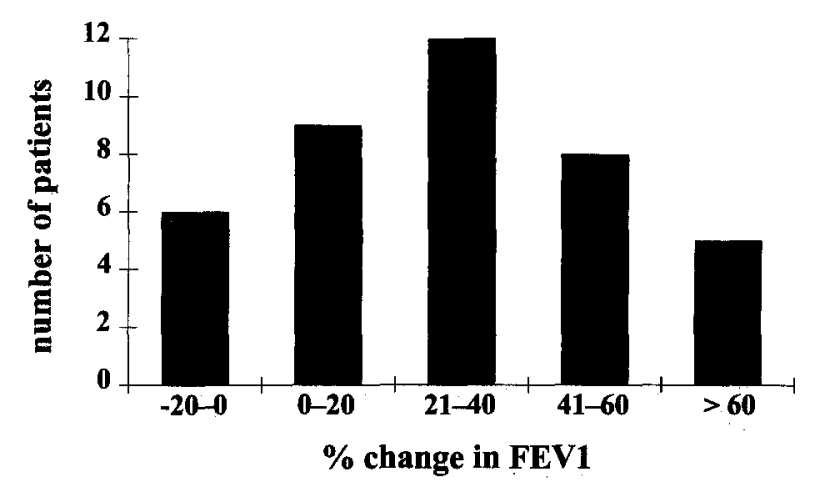

Fig. 2. Histogram demonstrating the range of improvement in postoperative $\mathrm{FEV}_{1}$ demonstrated by patients at 3-month evaluation.

ble overdistention of the remaining lung, chest tubes were placed on only $-10 \mathrm{~cm} \mathrm{H}_{2} \mathrm{O}$ of suction. After 24 hours, adjustments were made as necessary.

Patients were maintained on ventilatory assistance as needed. Our aim, however, was to achieve early postoperative extubation and independent ventilation as soon as possible after operation.

The incidences of postoperative complications were documented. Complications assessed included death, pneumonia, duration of air leaks, empyema, respiratory failure requiring prolonged ventilation, and cardiovascular complications:

Statistics and analysis. Data are expressed a mean \pm standard error of the mean. Student's $t$ tests for paired samples were used to examine changes in spirometry measurements, lung volumes, arterial blood gases, and 6-minute walk results. Fisher's Exact Test and $\chi^{2}$ tests were used to examine the differences between risk groups. Results were considered significant for $p$ less than 0.05 .

\section{Results}

There were 24 female and 43 male patients, with an average age of $61.9 \pm 8.1$ years (range 36 to 78 years). Operative procedure was pure laser ablation in 10 patients (laser group). The stapler group comprised the following procedures: stapled resection in 23 patients and stapler resection with modest laser ablation in 34 patients. In the latter group, less than $2500 \mathrm{~J}$ laser energy was used in any single case. Fifty-nine of the 67 patients were extubated in the operating room or within 2 hours of arrival in the intensive care unit. The remaining patients were extubated between 2 and 60 days after operation.

Laser group. Analysis of the patients in the laser group revealed that six of the 10 patients in whom the laser was the predominant operating tool had adverse outcomes. Two patients died in the postoperative period, and four additional patients had hospitalizations longer than 30 days for cardiopulmonary complications. This experience led us to
Table II. Complications of stapler or stapler-laser lung reduction surgery

\begin{tabular}{lc}
\multicolumn{1}{c}{ Complication } & No. of patients \\
\hline Air leak $>5$ days & 31 \\
Ventilation $>72 \mathrm{hr}$ & 7 \\
Conversion to open thoracotomy & 6 \\
Dysrhythmias & 4 \\
Empyema & 3 \\
Pneumonia & 2 \\
Stroke & 1 \\
Ischemic colitis & 1 \\
\hline
\end{tabular}

abandon the laser ablation technique in favor of stapled resection. The patients in the laser group are not included in the remaining analyses except for the analysis of predictors of poor outcome.

Stapler group. Among the 57 patients in the stapler group, there were two postoperative deaths $(3.4 \%)$. In addition there were three late deaths (longer than 4 months after operation), one each from pneumonia, stroke, and myocardial infarction. Four patients, including one of those accounting for the late deaths, had prolonged hospitalizations, ranging from 41 to 139 days.

Length of hospital stay averaged $17 \pm 11.1$ days. The most common complication was air leakage lasting beyond 5 days (Table II). The average duration of chest tube placement was $13 \pm 9.4$ days, with a range of 3 to 53 days. Intraoperative air leakage necessitated immediate conversion of the procedure to open thoracotomy in six patients. Three patients had postoperative empyemas, all of which were successfully managed with chest tube drainage.

Among the survivors, follow-up testing was performed at 3 months on 40 patients. Of the remaining 12 patients, five are scheduled for return visits at 6 months because of travel distance, one patient who had a postoperative stroke was not able to complete the full testing, one patient was found to have an unrelated cancer and is undergoing therapy, and one patient is not available for follow-up. Four other patients have refused 3-month follow-up testing.

Physiologic studies. The mean preoperative $\mathrm{FEV}_{1}$ was $0.82 \mathrm{~L}$, representing $30 \%$ of the predicted value. At 3-month follow-up, the mean $\mathrm{FEV}_{1}$ had risen significantly $(p<0.0001)$, to $1.04 \mathrm{~L}$ or $39 \%$ of predicted (Table III). Thirty-four patients (85\%) had improvement from preoperative values, with an average increase of $38 \%$. Fourteen patients $(35 \%)$ demonstrated improvements of greater than $40 \%$, ranging from $40 \%$ to $149 \%$, whereas six patients $(15 \%)$ showed no benefit from the procedure (Fig. 2). Similar improvements in FVC were noted after 
Table III. Changes in spirometry and lung volume measurements after lung reduction surgery

\begin{tabular}{|c|c|c|c|c|c|}
\hline & \multicolumn{2}{|c|}{ Before operation } & \multicolumn{2}{|c|}{3 months } & \multirow[b]{2}{*}{$p$} \\
\hline & Value & $\%$ Predicted & Value & $\%$ Predicted & \\
\hline $\mathrm{FEV}_{1}(\mathrm{~L})$ & $0.82 \pm 0.05$ & 30 & $1.04 \pm 0.06$ & 39 & $<0.0001$ \\
\hline FVC (L) & $2.26 \pm 0.14$ & 58 & $2.69 \pm 0.15$ & 70 & $<0.0001$ \\
\hline TLC (L) & $7.9 \pm 0.3$ & 139 & $7.4 \pm 0.3$ & 129 & $<0.0001$ \\
\hline $\mathrm{RV}(\mathrm{L})$ & $5.1 \pm 0.2$ & 239 & $4.3 \pm 0.2$ & 202 & $<0.0001$ \\
\hline RV/TLC ratio & 0.64 & & 0.58 & & $<0.0001$ \\
\hline DLCO & $38.1 \pm 2.5$ & & $42.4 \pm 2.2$ & & 0.018 \\
\hline
\end{tabular}

Data mean \pm SEM.

the lung reduction procedure. TLC, functional residual capacity, and RV all showed significant ( $p<$ 0.0001 ) declines after operation (Table II). The magnitude of the fall in RV, however, was greater than the reduction in TLC, as indicated by the RV/TLC ratio, which declined from 0.64 before operation to $0.58(p<0.0001)$ at 3 months after operation. The DLCO was also significantly improved after operation, from $38 \%$ before operation to $42 \%$ after operation $(p=0.018)$.

The room air oxygen tension did not change after operation; it was $64 \mathrm{~mm} \mathrm{Hg}$ before and $65 \mathrm{~mm} \mathrm{Hg}$ at 3 months after operation. There was a slight but significant $(p=0.034)$ decline in carbon dioxide tension $\left(\mathrm{PCO}_{2}\right)$, from $43 \pm 7 \mathrm{~mm} \mathrm{Hg}$ before operation to $40 \pm 8 \mathrm{~mm} \mathrm{Hg}$ at 3 months after operation. This improvement was more dramatic in the 12 patients who initially had hypercapnia $\left(\mathrm{PCO}_{2}>45\right.$ $\mathrm{mm} \mathrm{Hg}$ ). In this subgroup of patients, whose $\mathrm{PCO}_{2}$ ranged from 46 to $62 \mathrm{~mm} \mathrm{Hg}$, the mean $\mathrm{PCO}_{2}$ fell from 50.5 to $45 \mathrm{~mm} \mathrm{Hg}$ after operation $(p=0.018)$.

Functional measures. After operation, patients demonstrated functional improvement by walking further in the 6-minute walk test. Mean walk distance rose from $784 \pm 51$ feet before operation to $894 \pm 49$ feet at 3-month testing $(p=0.002)$. Subjective estimates of functional impairment by means of the preoperative baseline dyspnea index averaged 1.3 , indicating severe impairment. Three months after operation patients indicated a moderate improvement of symptoms, with a mean TDI of 1.7. Assessment of the TDI provided a method to determine whether there were any subjective differences between the patients who returned for 3 -month evaluation and those who did not. Telephone interviews conducted with the latter group revealed a mean TDI score of 1.4 , which was not significantly different from that of the returning cohort of patients.

The need for supplemental oxygen was eliminated in $17 \%$ of patients at 3-month follow-up. An addi- tional $25 \%$ of patients had reduced oxygen requirements during exercise.

Predictors of poor outcome. An interim analysis of the first 42 patients, including all 10 who underwent laser ablation only and the first 32 patients to undergo stapler or stapler-laser procedures, was performed to determine whether any preoperative variables were predictive for adverse outcome after lung reduction. An adverse outcome was defined as in-hospital death or as hospitalization longer than 30 days for cardiopulmonary complications. Ten patients demonstrated hypercapnia $\left(\mathrm{PCO}_{2} \geq 50 \mathrm{~mm}\right.$ $\mathrm{Hg}$ ) on the preoperative arterial blood gas analysis; six of these patients $(60 \%)$ had poor outcome, compared with only $17.9 \%(5 / 28)$ of the remaining evaluable patients $(p=0.0193)$. A similar number of patients (10) demonstrated a reduced $(\leq 25 \%)$ DLCO; again, the risk of a poor outcome was significantly higher $(p=0.0193)$ among these subjects $(60 \%)$ than among patients without this risk factor. Of particular importance was the finding that the combination of hypercapnia and reduced DLCO was $86 \%$ specific (five of six patients) in predicting serious postoperative risk ( $p=0.0026$ ). All subsequent patients referred for unilateral thoracoscopic lung reduction were not operated on if they had this combination of characteristics.

\section{Discussion}

The traditional indication for bullectomy, removal of dominant bullae with reexpansion of previously compressed lung tissue, has been emphasized by several authors., 16 The advent of video-assisted thoracic surgical techniques, in combination with laser thoracoscopy, led to application of laser ablation for both large and more diffuse bullous disease. ${ }^{6,17}$ Interpretation of the results of these series with respect to the value of such therapy for patients with diffuse emphysema remained difficult, however, because of the lack of objective data documenting improvement. Recently, the pioneering concepts of 
Brantigan, Kress, and Mueller ${ }^{3}$ have been resurrected, with a median sternotomy technique used to achieve bilateral lung reduction for patients with diffuse emphysema. ${ }^{8}$ This study documented the effectiveness of multiple wedge resection to produce both symptomatic (dyspnea index) and objective (spirometry, 6-minute walk) improvement in patients with end-stage emphysema refractory to medical management. It remained uncertain, however, whether a less invasive video-assisted approach could achieve comparable functional results. The focus of this study was to evaluate the unilateral thoracoscopic procedure for the treatment of patients with diffuse emphysema.

Our initial enthusiasm for the use of laser ablation to achieve lung reduction quickly became tempered by the discouraging clinical results. Six of the 10 patients had poor outcome, leading us to abandon the laser, except for minor portions of some procedures, in favor of stapled resection of strips of the most severely damaged lung. CT scan with density mask imaging to highlight the boundary zones between useful and emphysematous tissue, and the quantitative ventilation-perfusion scans with SPECT perfusion scintigraphy are crucial in identifying the areas to be resected. With these techniques as a guide, strips of diseased tissue are resected to reduce the volume of the lung by approximately $25 \%$ in a way that also tailors the resection to avoid space problems at the end of the case.

Air leakage requiring chest tube placement for longer than 5 days was the single most important complication, affecting 31 of the 57 patients undergoing stapled resection. Use of bovine pericardial strips to buttress the staple lines, as advocated by Cooper, ${ }^{18}$ has only recently become practical with sets designed for the endoscopic stapling devices. Nevertheless, most air leaks sealed within 2 weeks, and mean length of stay in the hospital (17 days) was similar to that reported after median sternotomy. ${ }^{8}$

Acceptance of any technique requires demonstration of objective improvement as well as subjective benefit. Spirometric values $\left(\mathrm{FEV}_{1}, \mathrm{FVC}\right)$ significantly increased after unilateral thoracoscopic lung reduction. In one third of the patients, the extent of improvement was in the range achieved with the bilateral approach. Lung volume measurements all fell after operation, with a disproportionate decline in RV compared with TLC. An improvement in inspiratory capacity and therefore FVC was the net result of this change. Functional progression in the ability to exercise was demonstrated by the signifi- cant improvement in the distances achieved during the 6-minute walk test. Finally, the subjective benefit of the operation was suggested by the significant improvement in functional impairment, as documented by the TDI. This level of improvement meant a return to most activities of daily living for the majority of patients.

Potential mechanisms for improvement after lung reduction procedures include increases in intrinsic elastic recoil and improvement in diaphragmatic function. Loss of elastic recoil, resulting in decreased alveolar driving pressure and increased expiratory resistance, leads to a reduction in the expiratory flow rate. With time, these changes lead to an increase in lung volumes as expiration is halted from premature airway collapse. These expanded lung volumes lead to "bowing" of the diaphragm and to inadequate generation of inspiratory contractile forces of intercostal and diaphragmatic muscles. Improvement in elastic recoil would increase the driving pressure that generates flow and would also reduce airway resistance by tethering the airways. Similarly, improved respiratory muscle efficiency should result from a reduction in end-expiratory lung volumes. Of note, in this study the decline in RV was greater than the change in TLC. Under these conditions, lung reduction surgery is not "volume reduction" because TLC declines only slightly. The improvement in the RV/TLC ratio results from significant increases in inspiratory capacity and expiratory reserve volume in the setting of a relatively fixed thoracic cage. It is likely that $\mathrm{FEV}_{1}$, an unnatural maneuver requiring maximal effort, does not reflect the entire spectrum of improvement resulting from improved elastic recoil and decreased lung volumes.

Finally, this study was undertaken to enhance our understanding of the preoperative selection criteria that best identify the most appropriate candidates. Candidates should have evidence of hyperinflation on a chest roentgenogram, with well-demarcated areas of trapped air or dead space determined by CT scan and ventilation-perfusion SPECT scanning. These areas should be associated both with wash-in and washout abnormalities and with hypoperfusion. In addition, we determined that the combination of significant resting hypercapnia $\left(\mathrm{PCO}_{2}>50 \mathrm{~mm} \mathrm{Hg}\right)$ and DLCO less than $25 \%$ of predicted was associated with an adverse outcome in five of six affected patients. Such patients should not be considered for unilateral thoracoscopic lung reduction.

Lung reduction procedures have the potential to 
benefit a large group of patients with end-stage diffuse emphysema who have exhausted their medical alternatives. These are patients for whom improved quality of life is as important as, if not more important, than the opportunity to extend life.

\section{REFERENCES}

1. American Lung Association. Facts about emphysema. New York: American Lung Association, 1990.

2. Connolly JE, Wilson A. The current status of surgery for bullous emphysema. J THORAC CARdrovasc SURG 1989;97:351-61.

3. Brantigan OC, Kress MB, Mueller EA. The surgical approach to pulmonary emphysema. Dis Chest 1961; 39:485-501.

4. Hazelrigg, SR, Nunchuck SK, LoCicero J. Video Assisted Thoracic Surgery Study Group data. Ann Thorac Surg 1993;56:1039-44.

5. Lewis RJ, Caccavale RJ, Sisler G. VATS-argon beam coagulator treatment of diffuse end-stage bilateral bullous disease of the lung. Ann Thorac Surg 1993; 55:1394-9.

6. Wakabayashi A, Brenner M, Kayaleh RA, et al. Thoracoscopic carbon dioxide laser treatment of bullous emphysema. Lancet 1991;337:881-3.

7. Barker SJ, Clarke C, Trivedi N, et al. Anesthesia for thoracoscopic laser ablation of bullous emphysema. Anesthesiology 1993;78:44-50.

8. Cooper JD, Trulock EP, Triantafillou AN, et al. Bilateral pneumectomy (volume reduction) for chronic obstructive pulmonary disease. J THORAC CARDIOVASC SURG 1995;109:106-19.

9. Morris JF. Spirometry in the evaluation of pulmonary function: medical progress. West J Med 1976;125: 110-8

10. Burrows B, Kasik JE, Niden AH, Barclay WR. Clinical usefulness of the single breath pulmonary diffusing capacity test. Am Rev Respir Dis 1961;84:789-806.

11. Goldman HT, Becklake MR. Respiratory function tests: normal values at median altitudes and the prediction of normal results. Am Rev Tuberc 1959; 79:457-67.

12. Muller NL, Staples CA, Miller RR, Abboud RT. "Density mask": an objective method to quantitate emphysema using computed tomography. Chest 1988; 94:782-7.

13. Kinsella M, Muller NL, Abboud RT, et al. Quantitation of emphysema by computed tomography using a "density mask" program and correlation with pulmonary function test. Chest 1990;97:315-21.

14. Wallis JW, Miller TR. Volume rendering in threedimensional display of SPECT images. J Nucl Med 1990;31:1421-30.

15. Mahler DA, Faryniarz K, Tomlinson D, et al. Impact of dyspnea and physiologic function on general health status in patients with chronic obstructive pulmonary disease. Chest 1991;102:395-401.

16. Gaensler EA, Cugell DW, Knudson RJ, FitzGerald MX. Surgical management of emphysema. Clin Chest Med 1983;4:443-63.

17. Brenner M, Kayaleh RA, Milne EN, et al. Thoracoscopic laser ablation of pulmonary bullae: radiographic selection and treatment response. J THORAC CaRdiovasC Surg 1994;107:883-90.

18. Cooper JD. Technique to reduce air leaks after resection of emphysematous lung. Ann Thorac Surg 1994;57:1038-9.

\section{Discussion}

Dr. Alec G. Patterson (St. Louis, Mo.). I am only substituting for the inevitable commentary that would have been provided by Joel Cooper had he been here. I compliment you on the work. It was a clear presentation and a lot of interesting data. Those SPECT images are fantastic. In how many cases have you used that imaging strategy?

Dr. Keenan. Probably about 40 now. We began to use it back in the late summer, and since that time it has been a major factor in our assessment.

Dr. Patterson. We have exclusively used a bilateral approach, except in patients with a specific contraindication to a bilateral operation such as previous operation or radiation. I think our results are approximately 50\% better than yours. One might expect this difference, given the improvement observed after bilateral versus unilateral procedures. Are there patients you consider should undergo a bilateral operation. If so, what is your current thought on such indications?

Dr. Keenan. I agree that the bilateral approach is of particular benefit for many patients. Of the total number of patients on whom we have performed lung reduction operations, we have done probably a dozen or so median sternotomies and not quite that many bilateral thoracoscopic approaches. I think the patients that would benefit from a bilateral approach from the beginning are those with extremely severe evidence of obstructive disease, perhaps the ones who have $\mathrm{FEV}_{1} \mathrm{~s}$ of $10 \%$ to $15 \%$, as opposed to $15 \%$ to $25 \%$. I think also that patients who have clearly symmetric disease with large areas on both sides, particularly both upper lobes, that can be attacked at the same time would benefit from a bilateral approach right away, as opposed to a unilateral approach. Those are the patients that we are now looking at as predominantly wanting to do bilateral approaches on.

We happen to want to explore the whole issue of bilateral thoracoscopic approaches, so we do the procedure as a thoracoscopy, flip the patient over, and do another thoracoscopy. Thoracoscopy offers a particularly good view of the entire hemithorax, as you know, and there may be some advantage in certain people for bilateral thoracoscopy as opposed to sternotomy. So I think that there will be a group of people, probably the worst-off patients, who should undergo the bilateral approach-no matter how it is performed-as the first step. 
I think that many others will obtain not just objective but symptomatic improvement with the unilateral approach. Perhaps one of the advantages might be that, with further follow-up and rehabilitation, you can then maybe carry the patients out a little bit further and then operate on the other side and carry them out again.

Dr. David Johnstone (Rochester, N.Y.). Since you have done a fair amount of thoracoscopic emphysema surgery and now some operations with the sternal approach, how do you decide how much lung to resect, and how do you assess whether you are going to have an air space? Because reexpansion of the lung with the video-assisted thoracoscopic surgical approach obliterates your view, I do not see how you reassess it during the operation.

Dr. Keenan. There has been a lot of discussion on how much lung you should resect. People have put arbitrary values-25\%,30\%- on it. I am not sure how you decide. Other people have looked at lung weights, saying you should take a certain number of grams of lung tissue. Clearly, that is going to be tremendously different between patients with air sacs versus those with a diffuse emphysema that has some substance to it. My approach is to go exactly where the SPECT imaging tells me I should go, and I do not go anywhere else. I also try to avoid the temptation to keep going, always doing a little bit more, and try to just restrict myself to what I decided I was going to do before operation. It is possible to get some idea of the potential for a space problem by periodic reinflation, one breath, two breaths at most, just to start seeing that it is going to fill up in the areas that you have previously resected. By using an endoscopic lung retractor, you can get a sense of whether there is a potential for development of a space problem. That is what I do. 